\title{
The Study on Hybridized Two-Band Superconductor
}

\author{
T. Chanpoom, ${ }^{1,2}$ J. Seechumsang, ${ }^{2,3}$ S. Chantrapakajee, ${ }^{4}$ and P. Udomsamuthirun ${ }^{2,3}$ \\ ${ }^{1}$ Program of Physics and General Science, Faculty of Science and Technology, Rajabhat Nakhon Ratchasima University, Thailand \\ ${ }^{2}$ Prasarnmitr Physics Research Unit, Department of Physics, Faculty of Science, Srinakharinwirot University, Sukhumvit 23, \\ Bangkok 10110, Thailand \\ ${ }^{3}$ Thailand Center of Excellence in Physics (ThEP), Si Ayutthaya Road, Bangkok 10400, Thailand \\ ${ }^{4}$ Rajamangala University of Technology Phra Nakhon, 399 Samsen Road, Dusit, Bangkok 10300, Thailand
}

Correspondence should be addressed to P. Udomsamuthirun; udomsamut55@yahoo.com

Received 3 December 2012; Revised 8 March 2013; Accepted 22 March 2013

Academic Editor: Victor V. Moshchalkov

Copyright (C) 2013 T. Chanpoom et al. This is an open access article distributed under the Creative Commons Attribution License, which permits unrestricted use, distribution, and reproduction in any medium, provided the original work is properly cited.

\begin{abstract}
The two-band hybridized superconductor which the pairing occurred by conduction electron band and other-electron band are considered within a mean-field approximation. The critical temperature, zero-temperature order parameter, gap-to- $T_{c}$ ratio, and isotope effect coefficient are derived. We find that the hybridization coefficient shows a little effect on the superconductor that conduction electron band has the same energy as other-electron band but shows more effect on the superconductor that conduction electron band coexists with lower-energy other-electron band. The critical temperature is decreased as the hybridization coefficient increases. The higher value of hybridization coefficient, lower value of gap-to- $T_{c}$ ratio, and higher value of isotope effect coefficient are found.
\end{abstract}

\section{Introduction}

Since Moskalenko [1, 2] Suhl et al. [3] introduced the twoband model that accounts for multiple energy bands in the vicinity of the Fermi energy contributing electron pairing in superconductor, the two-band model has been applied to high temperature superconductor in copper oxides [4-10], $\mathrm{MgB}_{2}$ superconductor [11-13], and heavy Fermion superconductor $[14,15]$. Dolgov et al. [16] studied the thermodynamic properties of the two-band superconductor: $\mathrm{MgB}_{2}$. The superconducting energy gap, free energy, the entropy, and heat capacity were calculated within the framework of twoband Eliashberg theory. Mazin et al. [17] studied the effect of interband impurity scattering on the critical temperature of two-band superconductor in $\mathrm{MgB}_{2}$. Askerzade and Tanatar [18] and Changjan and Udomsamuthirun [19] calculated the critical field of the two-band superconductor by GinzburgLandau approach and applied it to Fe-based superconductors. Golubov and Koshelev [20] investigated the two-band superconductor with strong intraband and weak interband electronic scattering rates in the framework of coupled Usadel equation.
The interplay of superconductivity and magnetism is one of the most interesting phenomena of superconductor. The cuprate superconductor exhibits the phase diagram having the magnetic ordered states in the vicinity of the superconducting phase. The antiferromagnetic and ferromagnetic phases are also found in the heavy Fermion superconductor. In $\mathrm{ErRh}_{4} \mathrm{~B}_{6}$ [21] and $\mathrm{HoMo}_{6} \mathrm{~S}_{8}$ [22] system, $s$-wave superconductivity shows ferromagnetism in the ground state at intermediate temperature.

The nesting properties of Fermi surface in low dimensional system arise the spin density wave (SDW) state and charge density wave (CDW) state in the interplay of superconductivity and magnetism system. The SDW and the CDW states occurred by Coulomb interaction between electron, and electron-phonon interaction, respectively. Nass et al. [23] used the BCS-type pairing to explain the antiferromagnetic superconductor. Suzumura and Nagi [24] investigated some properties of antiferromagnetic superconductor. They proposed the Hamiltonian of the superconductivity associated with conduction $d$-electron and the antiferromagnetism associated with $f$-electron of rare earth atoms that formed the BCS-type pairing. Ichimura et al. [25] investigated the effect 
of the CDW on BCS superconductor within a mean-field approximation. In this model, the two order parameters of CDW and superconductor were introduced. The tightbinding band in $2 \mathrm{D}$ square lattice and nesting vector $\mathrm{Q}=$ $(\pi, \pi)$ was used in their calculations. Rout and Das [26] applied the periodic Anderson model (PAM) for calculating the nonmagnetic ground state of heavy Fermion superconductor. The Hamiltonian of the heavy Fermion systems composed of conduction electron band, $f$-electron band, the hybridization of conduction electron and $f$-electron band, BCS-like pairing band, and the intra-atomic Coulomb interaction of $f$-electron. The Hamiltonian was simplified by linearising the intra-atomic Coulomb interaction with the Hartree-Fock approximation then the $f$-electron band energy was $E_{k}=\varepsilon_{f}+U n_{\sigma}$, where $\varepsilon_{f}$ was bare $f$-electron energy and $U n_{\sigma}$ was the Coulomb energy of $f$-electron. Finally, their Hamiltonian were consisted of conduction and $f$-electron band including BCS-like pairing band and hybridization term. Panda and Rout [27] studied the interplay of CDW, SDW, and superconductivity in high temperature superconductor in low doping phase. The model of meanfield Hamiltonian including the CDW, SDW and superconductivity was introduced.

In this paper, we modified the hybridized Hamiltonian of Rout and Das [26] to be the two-band superconductor with hybridization. Some physical properties of two-band hybridized superconductor, that is, critical temperature, zerotemperature order parameter, gap-to- $T_{c}$ ratio, and isotope effect coefficient, were investigated.

\section{Model and Calculation}

According to the hybridized Hamiltonian [26] that consisted of conduction electron and $f$-electron band, BCS-like pairing band, and hybridization term, we have

$$
\begin{aligned}
H= & \sum_{k, \sigma} \varepsilon_{k} C_{k \sigma}^{+} C_{k \sigma}+\varepsilon_{f} \sum_{k, \sigma} f_{k \sigma}^{+} f_{k \sigma}+\gamma_{0} \sum_{k, \sigma}\left(f_{k \sigma}^{+} C_{k \sigma}+C_{k \sigma}^{+} f_{k \sigma}\right) \\
& +\frac{U}{2} \sum_{i, \sigma} n_{i \sigma}^{f} n_{i,-\sigma}^{f}-\Delta \sum_{k}\left(C_{k \uparrow}^{+} C_{-k \downarrow}^{+}+C_{-k \downarrow} C_{k \uparrow}\right),
\end{aligned}
$$

where $C_{k \sigma}^{+}\left(C_{k \sigma}\right)$ and $f_{k \sigma}^{+}\left(f_{k \sigma}\right)$ are the creation (annihilation) operator of conduction electron and $f$-electron. $\Delta$ is the superconducting order parameter that Cooper pairs involve only the conduction electron. $\gamma_{0}$ is the hybridization interaction coefficient of $f$-electron band and conduction band. $n_{i}^{f}=f_{i \sigma}^{+} f_{i \sigma}$ is the intraatomic Coulomb interaction between $f$-electron. They [26] linearised the $(U / 2) \sum_{i, \sigma} n_{i \sigma}^{f} n_{i,-\sigma}^{f}$ term by Hartree-Fock approximation that $(U / 2) \sum_{i, \sigma} n_{i \sigma}^{f} n_{i,-\sigma}^{f} \approx$ $U \sum_{i, \sigma} n_{-\sigma} f_{i \sigma}^{+} f_{i \sigma}$; then the Hamiltonian became

$$
\begin{aligned}
H= & \sum_{k, \sigma} \varepsilon_{k} C_{k \sigma}^{+} C_{k \sigma}+\sum_{k, \sigma} E_{0} f_{k \sigma}^{+} f_{k \sigma}+\gamma_{0} \sum_{k, \sigma}\left(f_{k \sigma}^{+} C_{k \sigma}+C_{k \sigma}^{+} f_{k \sigma}\right) \\
& -\Delta \sum_{k}\left(C_{k \uparrow}^{+} C_{-k \downarrow}^{+}+C_{-k \downarrow} C_{k \uparrow}\right),
\end{aligned}
$$

where $E_{0}=\varepsilon_{f}+U n_{-\sigma}$ that is the energy collected the noninteraction with a modified $f$-level.

In our model, the two-band superconductor comprise of conduction electron and other-electron band. The superconducting order parameters can occurr by conduction electron and other-electron band. The conduction band makes the intra-atomic Coulomb interaction with other-electron band. We set

$$
\begin{aligned}
H= & \sum_{k, \sigma} \varepsilon_{k} C_{k \sigma}^{+} C_{k \sigma}+\sum_{k, \sigma} E_{0} f_{k \sigma}^{+} f_{k \sigma}+\gamma_{0} \sum_{k, \sigma}\left(f_{k \sigma}^{+} C_{k \sigma}+C_{k \sigma}^{+} f_{k \sigma}\right) \\
& +\frac{U}{2} \sum_{i, \sigma} n_{i \sigma}^{f} n_{i,-\sigma}^{f}-\Delta \sum_{k}\left(C_{k \uparrow}^{+} C_{-k \downarrow}^{+}+C_{-k \downarrow} C_{k \uparrow}\right) \\
& -\Delta \sum_{k}\left(f_{k \uparrow}^{+} f_{-k \downarrow}^{+}+f_{-k \downarrow} f_{k \uparrow}\right),
\end{aligned}
$$

where $C_{k \sigma}^{+}\left(C_{k \sigma}\right)$ and $f_{k \sigma}^{+}\left(f_{k \sigma}\right)$ are the creation (annihilation) operators of conduction electron and other-electron band. $\Delta$ is the superconducting order parameter. $\gamma_{0}$ is the hybridization interaction coefficient of other-electron band and conduction band. $n_{i}^{f}=f_{i \sigma}^{+} f_{i \sigma}$ is the intra-atomic Coulomb interaction between other-electron. The Hamiltonian is linearised by [26]'s technique; then we get the simplified two-bandhybridized Hamiltonian. We can write the Hamiltonian as

$$
H=H_{1}+H_{2}+H_{12}
$$

where

$$
\begin{gathered}
H_{1}=\sum_{k \sigma} \varepsilon_{k} C_{k \sigma}^{+} C_{k \sigma}-\Delta \sum_{k}\left(C_{k \uparrow}^{+} C_{-k \downarrow}^{+}+C_{-k \downarrow} C_{k \uparrow}\right), \\
H_{2}=\sum_{k \sigma} E_{k} f_{k \sigma}^{+} f_{k \sigma}-\Delta \sum_{k}\left(f_{k \uparrow}^{+} f_{-k \downarrow}^{+}+f_{-k \downarrow} f_{k \uparrow}\right), \\
H_{12}=\gamma_{0} \sum_{k \sigma}\left(f_{k \sigma}^{+} C_{k \sigma}+C_{k \sigma}^{+} f_{k \sigma}\right) .
\end{gathered}
$$

The first Hamiltonian describes the conduction electron Hamiltonian, the second Hamiltonian describes the Hamiltonian of other-electron band, and the third Hamiltonian describes the interact Hamiltonian. Where $\varepsilon_{k}$ and $E_{k}$ are the band energies of the conduction electron and other-electron band measured from the Fermi energy.

$E_{k}=E_{0}+U n_{-\sigma}$ is the energy collected the non-interaction with a modified other-electron band. $C_{k \sigma}^{+}\left(C_{k \sigma}\right)$ and $f_{k \sigma}^{+}\left(f_{k \sigma}\right)$ are the creation (annihilation) operator of conduction electron and other-electron. $\gamma_{0}$ is the hybridization interaction coefficient of other-electron band and conduction band. $\Delta$ is the effective superconducting order parameter occurred by conduction electron and other-electron and assumed to be homogeneous in space. The effective superconducting order parameters is

$$
\Delta=\frac{V}{2} \sum_{k}\left(\left\langle C_{k \uparrow}^{+} C_{-k \downarrow}^{+}\right\rangle+\left\langle f_{k \uparrow}^{+} f_{-k \downarrow}^{+}\right\rangle\right),
$$

where the $s$-wave like BCS pairing interaction having the same coupling interaction potential is assumed. The effective 
superconducting order parameters are the coupling equation of conduction electron and other-electron that can occurr in magnetic superconductor [23-25]. However for simplicity of calculation, the same pairing strength is taken [28].

We introduce the finite-temperature Green function:

$$
G(k, \tau)=-\left\langle T_{\tau} \psi_{k}(\tau) \psi_{k}^{+}(0)\right\rangle,
$$

where $\psi_{k}^{+}=\left(C_{k \uparrow}^{+}, C_{-k \downarrow}, f_{k \uparrow}^{+}, f_{-k \downarrow}\right)$ and $T_{\tau}$ is the ordering operator for imaginary time, $\tau=i t$.

After some calculations, the Green function in Nambu representation is obtained:

$$
\begin{aligned}
G\left(\omega_{n}, k\right)=( & i \omega_{n}-\left(\frac{\varepsilon_{k}-E_{k}}{2}\right) \rho_{3} \sigma_{3}-\left(\frac{\varepsilon_{k}+E_{k}}{2}\right) \sigma_{3} \\
& \left.+\Delta \sigma_{1}-\gamma_{0} \rho_{1} \sigma_{3}\right)^{-1},
\end{aligned}
$$

where $\omega_{n}=\pi T(2 n+1), T$ is temperature, $n$ is an integer, and $\rho_{i}$ and $\sigma_{i}(i=1,2,3)$ are the Pauli matrices. Our Green function obtained shows the same form as [25] that investigated the effect of the CDW on BCS superconductor within a meanfield approximation, $G^{-1}\left(\omega_{n}, k\right)=i \omega_{n}-\gamma_{k} \rho_{3} \sigma_{3}-\delta_{k} \rho_{0} \sigma_{3}+$ $\Delta \rho_{0} \sigma_{1}+w \rho_{1} \sigma_{3}$. All parameters detailed can be found in [25]. We find that $\left(\varepsilon_{k}-E_{k}\right) / 2 \equiv \gamma_{k}$ and $\left(\varepsilon_{k}+E_{k}\right) / 2 \equiv \delta_{k}$, where $\gamma_{k}$ and $\delta_{k}$ are the band structure energies in 2D square lattice of nearest-neighbor and next-nearest-neighbor transfer, respectively. And $-\gamma_{0} \equiv w$ which $w$ is the order parameter of CDW. This result means that the CDW consideration gives the same result as the hybridization consideration within a mean-field approximation.

From (6) and (8), the superconducting gap equation is

$$
\begin{gathered}
\frac{1}{V}=\frac{1}{4} \sum_{k}\left(\frac{\tanh \left(\sqrt{\Delta^{2}+\varepsilon_{-}^{2}} / 2 T\right)}{\sqrt{\Delta^{2}+\varepsilon_{-}^{2}}}\right. \\
\left.+\frac{\tanh \left(\sqrt{\Delta^{2}+\varepsilon_{+}^{2}} / 2 T\right)}{\sqrt{\Delta^{2}+\varepsilon_{+}^{2}}}\right),
\end{gathered}
$$

where $\varepsilon_{+}=\left(\varepsilon_{k}+E_{k}\right) / 2+\sqrt{\left(\left(\varepsilon_{k}-E_{k}\right) / 2\right)^{2}+\gamma_{0}^{2}}$ and $\varepsilon_{-}=$ $\left(\varepsilon_{k}+E_{k}\right) / 2-\sqrt{\left(\left(\varepsilon_{k}-E_{k}\right) / 2\right)^{2}+\gamma_{0}^{2}}$. The $\varepsilon_{+}$and $\varepsilon_{-}$represent the upper and lower bands of quasiparticle energy spectra of the hybridization system. We can determine the superconducting critical temperature $T_{c}$ by putting $\Delta \rightarrow 0$; then

$$
\frac{1}{V}=\frac{1}{4} \sum_{k}\left(\frac{\tanh \left(\varepsilon_{-} / 2 T_{c}\right)}{\varepsilon_{-}}+\frac{\tanh \left(\varepsilon_{+} / 2 T_{c}\right)}{\varepsilon_{+}}\right) .
$$

In the absence of the hybridization interaction, $\gamma_{0}=0$; that is,

$$
\frac{1}{V}=\frac{1}{2} \sum_{k}\left(\frac{\tanh \left(\varepsilon / 2 T_{c 0}\right)}{\varepsilon}\right) \text { or } \frac{1}{\lambda}=\ln \left(\frac{2 \gamma \omega_{D}}{\pi T_{c 0}}\right) \text {, }
$$

where $\gamma=1.78 . \lambda=N(0) V, \lambda$ is the coupling constant, and $N(0)$ is the constant density of state at the Fermi surface, and
$\omega_{D}$ is the Debye cutoff energy. $T_{c 0}$ is the critical temperature of superconductor without hybridization that the BCS's result.

Because of the complicated quasi-particle energy spectra obtained, we introduce the two approximated conditions to calculate analytically; the superconductor with conduction electron band having the same energy as other-electron band $\left(\varepsilon_{k} \approx E_{k}\right)$ and the superconductor with conduction electron band coexisting with lower-energy other-electron band $\left(\varepsilon_{k} \gg\right.$ $\left.E_{k}, E_{k} \approx 0\right)$.

Case 1. The superconductor that the conduction electron band having the same energy as other-electron band.

In this case, the approximation is $\varepsilon_{k} \approx E_{k}$. Then, we get $\varepsilon_{-} \approx \varepsilon_{k}-\gamma_{0}$ and $\varepsilon_{+} \approx \varepsilon_{k}+\gamma_{0}$. The difference of the lower and upper energy spectra is equal to $2 \gamma_{0}$. If $\gamma_{0}=0$, the BCS's superconductor is obtained. In this case, the effect of the hybridization interaction on the BCS superconductor is considered.

We substitute above approximations into (10); then the gap equation becomes

$$
\begin{aligned}
\frac{1}{\lambda} & =\frac{1}{4}\left(\int_{-\omega_{D}}^{\omega_{D}} \frac{\tanh \left(\varepsilon_{-} / 2 T_{c}\right)}{\varepsilon_{-}} d \varepsilon_{k}+\int_{-\omega_{D}}^{\omega_{D}} \frac{\tanh \left(\varepsilon_{+} / 2 T_{c}\right)}{\varepsilon_{+}} d \varepsilon_{k}\right) \\
& \approx \ln \left(\frac{2 \gamma \sqrt{\omega_{D}^{2}-\gamma_{0}^{2}}}{\pi T_{c}}\right) .
\end{aligned}
$$

The critical temperature is

$$
T_{c}=1.13 \sqrt{\omega_{D}^{2}-\gamma_{0}^{2}} e^{-1 / \lambda}
$$

And the zero-temperature energy gap can be found as

$$
\begin{gathered}
\frac{1}{\lambda}=\frac{1}{4} \int_{-\omega_{D}}^{\omega_{D}}\left(\frac{1}{\sqrt{\Delta^{2}(0)+\left(\varepsilon_{k}-\gamma_{0}\right)^{2}}}\right) d \varepsilon_{k} \\
\left.+\frac{1}{\sqrt{\Delta^{2}(0)+\left(\varepsilon_{k}+\gamma_{0}\right)^{2}}}\right) \\
=\frac{1}{2}\left(\sinh h^{-1}\left(\frac{\omega_{D}-\gamma_{0}}{\Delta(0)}\right)+\sinh ^{-1}\left(\frac{\omega_{D}+\gamma_{0}}{\Delta(0)}\right)\right) .
\end{gathered}
$$

For $\omega_{D} \gg \Delta(0)$, we can get

$$
\Delta(0)=2 \sqrt{\omega_{D}^{2}-\gamma_{0}^{2}} e^{-1 / \lambda}
$$

From (13) and (15), the gap-to- $T_{c}$ ratio is obtained:

$$
R=\frac{2 \Delta(0)}{T_{c}}=3.53 \text {. }
$$

In this case, we find that the hybridization interaction coefficient decreases the critical temperature and zero-temperature energy gap but has no effect on gap-to- $T_{c}$ ratio. 
To investigate the effect of hybridization and the Debye cutoff on gap-to- $T_{c}$ ratio, we rewrite the gap equation at critical temperature and at zero-temperature into the form [29]

$$
\int_{\left(-\omega_{D}+\gamma_{0}\right) /\left(2 T_{c}\right)}^{\left(\omega_{D}+\gamma_{0}\right) /\left(2 T_{c}\right)} \frac{\tanh x}{x} d x=\int_{-2\left(\omega_{D}-\gamma_{0}\right) / T_{c}}^{2\left(\omega_{D}+\gamma_{0}\right) / T_{c}} \frac{1}{\sqrt{R^{2}+x^{2}}} d x .
$$

The numerical calculation of (17) is shown in Figure 2.

Within the definition of isotope effect coefficient in harmonic approximation; $\alpha=(1 / 2)\left(\omega_{D} / T_{c}\right)\left(d T_{c} / d \omega_{D}\right)$ and equation (9), we

$$
\alpha=\frac{\omega_{D}}{2}\left(\frac{\tanh \left(\omega_{D}^{-} / 2 T_{c}\right) / \omega_{D}^{-}+\tanh \left(\omega_{D}^{+} / 2 T_{c}\right) / \omega_{D}^{+}}{\tanh \left(\omega_{D}^{-} / 2 T_{c}\right)+\tanh \left(\omega_{D}^{+} / 2 T_{c}\right)}\right),
$$

where $\omega_{D}^{+}=\omega_{D}+\gamma_{0}$ and $\omega_{D}^{-}=\omega_{D}-\gamma_{0}$.

Consider the limiting cases that the hybridization is so small with respect to Debye cutoff energy, $\omega_{D} \gg \gamma_{0}$; for $\omega_{D}>$ $2 T_{c}$, we can get that $\alpha \approx\left(\omega_{D} / 4\right)\left(1 / \omega_{D}^{-}+1 / \omega_{D}^{+}\right) \approx 1 / 2$, and for $\omega_{D}<2 T_{c}$, we get $\alpha \approx\left(\omega_{D} / 2\right)\left(\left(1 / T_{c}\right) /\left(\omega_{D} / T_{c}\right)\right) \approx 1 / 2$ that the BCS' result.

Case 2. The superconductor that the conduction electron band coexisting with lower-energy other-electron band.

Because of the hybridization Hamiltonian having the same Green's function as the charge density wave model, we can apply this model to the superconducting state found in heavy Fermion superconductor. The heavy Fermion superconductor has its origin in the interplay of strong Coulomb repulsion in $4 \mathrm{f}$ - and $5 \mathrm{f}$-shells and their hybridizations with the conduction band. The $f$-electron is associated with the magnetic ordering having lower energy than conduction electron. We can make the assumption that the $f$-electron band is at the Fermi level which can be taken as $E_{k} \approx 0$; then

$$
\begin{gathered}
\varepsilon_{-} \approx \frac{\varepsilon_{k}}{2}-\gamma_{0}, \quad \varepsilon_{+} \approx \frac{\varepsilon_{k}}{2}+\gamma_{0}, \quad \text { for } \frac{\varepsilon_{k}}{2}<\gamma_{0}, \\
\varepsilon_{-} \approx 0, \quad \varepsilon_{+} \approx \varepsilon_{k}, \quad \text { for } \frac{\varepsilon_{k}}{2}>\gamma_{0} .
\end{gathered}
$$

Substituting above approximation into (10), we can get

$$
\begin{aligned}
\frac{1}{\lambda} & =\frac{1}{4}\left(\int_{-\omega_{D}}^{\omega_{D}} \frac{\tanh \left(\varepsilon_{-} / 2 T_{c}\right)}{\varepsilon_{-}} d \varepsilon_{k}+\int_{-\omega_{D}}^{\omega_{D}} \frac{\tanh \left(\varepsilon_{+} / 2 T_{c}\right)}{\varepsilon_{+}} d \varepsilon_{k}\right) \\
& \approx \ln \left(\frac{2 \gamma}{\pi T_{c}} \sqrt{2 \omega_{D} \gamma_{0}}\right)+\frac{1}{2}\left(\frac{\omega_{D}}{2 T_{c}}-\frac{\gamma_{0}}{T_{c}}\right),
\end{aligned}
$$

where,

$$
\begin{gathered}
\int_{-\omega_{D}}^{\omega_{D}} \frac{\tanh \left(\varepsilon_{-} / 2 T_{c}\right)}{\varepsilon_{-}} d \varepsilon_{k} \approx 2\left(\frac{\omega_{D}}{2 T_{c}}-\frac{2 \gamma_{0}}{2 T_{c}}\right)+2 \ln \left(\frac{4 \gamma \gamma_{0}}{\pi T_{c}}\right), \\
\int_{-\omega_{D}}^{\omega_{D}} \frac{\tanh \left(\varepsilon_{+} / 2 T_{c}\right)}{\varepsilon_{+}} d \varepsilon_{k} \approx 2 \ln \left(\frac{2 \gamma \omega_{D}}{\pi T_{c}}\right) .
\end{gathered}
$$

The critical temperature is

$$
T_{c}=1.13 \sqrt{2 \gamma_{0} \omega_{D}} e^{-1 / \lambda+(1 / 2)\left(\omega_{D} / 2 T_{c}-\gamma_{0} / T_{c}\right)} .
$$

The gap equation as zero-temperature is

$$
\frac{1}{\lambda}=\frac{1}{4} \int_{-\omega_{D}}^{\omega_{D}}\left(\frac{1}{\sqrt{\Delta^{2}(0)+\left(\varepsilon_{-}\right)^{2}}}+\frac{1}{\sqrt{\Delta^{2}(0)+\left(\varepsilon_{+}\right)^{2}}}\right) d \varepsilon_{k},
$$

where,

$$
\begin{aligned}
& \int_{-\omega_{D}}^{\omega_{D}} \frac{1}{\sqrt{\Delta^{2}(0)+\left(\varepsilon_{-}\right)^{2}}} d \varepsilon_{k} \\
& \approx\left(\frac{2}{\Delta(0)}\right)\left(\omega_{D}-2 \gamma_{0}\right)+2 \sinh ^{-1}\left(\frac{2 \gamma_{0}}{\Delta(0)}\right), \\
& \int_{-\omega_{D}}^{\omega_{D}} \frac{1}{\sqrt{\Delta^{2}(0)+\left(\varepsilon_{+}\right)^{2}}} d \varepsilon_{k} \approx 2 \sinh ^{-1}\left(\frac{\omega_{D}}{\Delta(0)}\right) .
\end{aligned}
$$

Then, we get

$$
\Delta(0)=2 \sqrt{2 \gamma_{0} \omega_{D}} e^{-1 / \lambda+(1 / 2)\left(\left(\omega_{D}-2 \gamma_{0}\right) / \Delta(0)\right)} .
$$

According to (22) and (25), the gap-to- $T_{c}$ ratio is

$$
R=\frac{2 \Delta(0)}{T_{c}}=3.53 e^{\left(\left(\omega_{D}-2 \gamma_{0}\right) / T_{c}\right)(1 / R-1 / 4)} .
$$

To investigate the effect of hybridization and the Debye cutoff on gap-to- $T_{c}$ ratio, we rewrite the gap equation at critical temperature and at zero-temperature into the form [29]

$$
\begin{array}{r}
\int_{-\omega_{D} / T_{c}}^{\omega_{D} / T_{c}} \frac{\tanh (y / 4-\sqrt{(y / 4)})}{x} d x \\
=\int_{-2\left(\omega_{D}-\gamma_{0}\right) / T_{c}}^{2\left(\omega_{D}+\gamma_{0}\right) / T_{c}} \frac{1}{\sqrt{R^{2}+x^{2}}} d x .
\end{array}
$$

The numerical calculation of this equation is shown in Figure 2.

Within the definition of isotope effect coefficient in harmonic approximation, (10) and $E_{k} \approx 0$, we can get

$$
\begin{aligned}
\alpha= & \left(\frac{\omega_{D}}{2}\right) \\
& \times\left(\frac{1+\left(2 T_{c} / \omega_{D}\right) \tanh \left(\omega_{D} / 2 T_{c}\right)}{\omega_{D}-2 \gamma_{0}+2 T_{c}\left(\tanh \left(\omega_{D} / 2 T_{c}\right)+\tanh \left(\gamma_{0} / T_{c}\right)\right)}\right) .
\end{aligned}
$$

\section{Results and Discussions}

We use the hybridized two-band Hamiltonian to investigate the critical temperature, zero-temperature order parameter, gap-to- $T_{c}$ ratio, and isotope effect coefficient of superconductor. The Green function and gap equation are derived analytically. However, the quasi-particle energy spectra obtained 


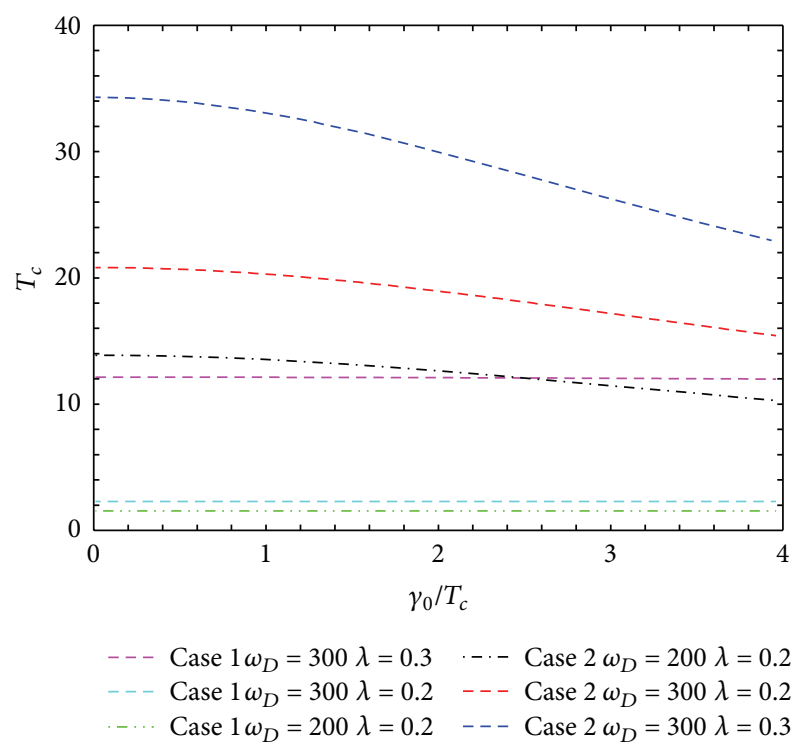

FIGURE 1: The $T_{c}$ versus hybridization coefficients of Cases 1 and 2.

are complicated; then we introduce two approximated cases as, the superconductor that conduction electron band has the same energy as other-electron band $\left(\varepsilon_{k} \approx E_{k}\right)$ and the superconductor that conduction electron band coexists with lower-energy other-electron band $\left(\varepsilon_{k} \gg E_{k}, E_{k} \approx 0\right)$. The critical temperature, zero-temperature order parameter, gapto- $T_{c}$ ratio, and isotope effect coefficient are shown in the exact forms.

We use the integration forms of involved equations for more accuracy in numerical calculation. After the numerical calculations of $T_{c}$, (Figure 1), we find that the weak-coupling limit $(\lambda<0.4)$ can be found in range of $\omega_{D} / T_{c}>10$. Then, we investigate the effect of hybridization in weak-coupling limit with $\omega_{D} / T_{c}>10$ and $\gamma_{0} / T_{c}=0.2,4.0$. We find that $T_{c}$ is decreased when the hybridization coefficient increased in Case 2 and no effect in Case 1. In Figure 2, the gap-to- $T_{c}$ ratios $(R)$ of Cases 1 and 2 with varied hybridization coefficients are shown. In Case 1 , the $R$ is tended to BCS value, 3.53, as $\omega_{D} / T_{c} \rightarrow \infty$. Case $2, R \approx 3.3-3.9$ can be found to depend strongly on the value of $\gamma_{0} / T_{c}$. The higher value of $\gamma_{0} / T_{c}$ and the lower value of $R$ are found to agree with superconducting gap's behavior of HF system of Rout et al. [30].

The isotope effect coefficient is also investigated and shown in Figure 3. In Case 2, we find that the isotope effect coefficient can be more than the BCS $(\alpha>0.5)$ and less than BCS value $(\alpha<0.5)$, but in Case 1 we can find only for $\alpha>0.5$. However, $\alpha$ in both cases is converse to 0.5 as $\omega_{D} / T_{c} \rightarrow \infty$.

The Fe-based superconductors are mutiband system which comprises at least two bands which propose $s$-wave paring state. The $R=3.68$ [31] which shows a consistent manner with the BCS prediction is found. The value of isotope effect coefficient were found to be $\alpha \approx 0.35-0.4$ [32] and $\alpha_{\mathrm{Fe}}=0.81$ [33]. These results indicate that electron-phonon interaction plays some role in the superconducting mechanism by affecting the magnetic properties [31]. According to our model, the effective superconducting order parameters

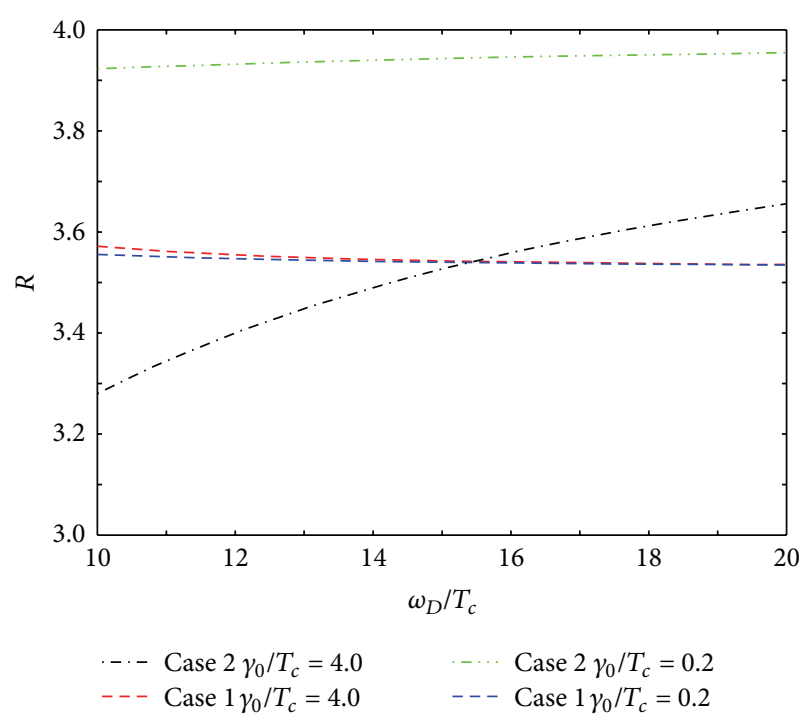

Figure 2: The gap-to- $T_{c}$ ratio of Cases 1 and 2 with varied hybridization coefficients.

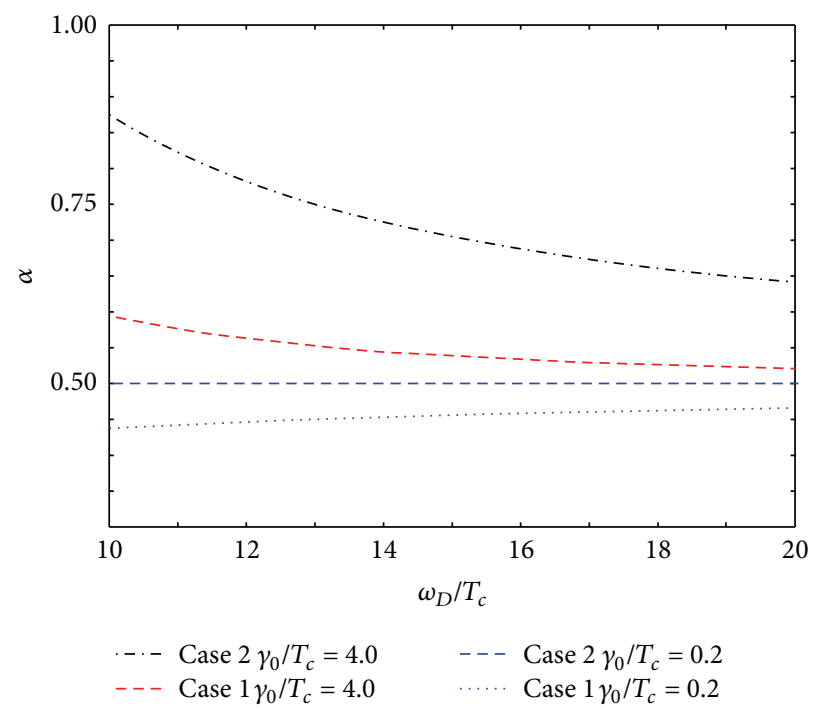

FIGURE 3: The isotope effect coefficient of Cases 1 and 2 with varied hybridization coefficients.

are the coupling equation of conduction electron and otherelectron and the magnetic order are included in our calculation as in Case 2. We can get the experimental data of Febased superconductor from Figures 2 and 3.

\section{Conclusions}

The two-band hybridized superconductor that the pairing occurred by conduction electron band and other-electron band is studied in weak-coupling limit. The formula of critical temperature, zero-temperature order parameter, gap-to- $T_{c}$ ratio and isotope effect coefficient are calculated. For the superconductor that conduction electron band has the same energy as other-electron band, the hybridization coefficient 
shows a little effect. The numerical results do not differ much from the BCS's results. For the superconductor that conduction electron band coexists with lower-energy other-electron band, the hybridization coefficient show more effect. $T_{c}$ is decreased when the hybridization coefficient increases. We can get higher and lower value of $R$ and $\alpha$ than BCS's results depending on the hybridization coefficient. Higher value of hybridization coefficient, lower value of $R$, and higher value of $\alpha$ are found.

\section{Acknowledgments}

The financial support of the Office of the Higher Education Commission, Srinakhariwirout University, and ThEP Center is acknowledged.

\section{References}

[1] V. A. Moskalenko, "Superconductivity of metals taking intoaccount the overlap of the energy bands," Fizika Metallov i Metallovedenie, vol. 8, p. 503, 1959.

[2] V. A. Moskalenko, Physics of Metals and Metallography, vol. 8, p. 25, 1959.

[3] H. Suhl, B. T. Matthias, and L. R. Walker, "Bardeen-cooperschrieffer theory of superconductivity in the case of overlapping bands," Physical Review Letters, vol. 3, no. 12, pp. 552-554, 1959.

[4] S. Robaszkiewicz, R. Micnas, and J. Ranninger, "Superconductivity in the generalized periodic Anderson model with strong local attraction," Physical Review B, vol. 36, no. 1, pp. 180-201, 1987.

[5] V. Z. Kresin and S. A. Wolf, "Multigap structure in the cuprates," Physica C, vol. 169, no. 5-6, pp. 476-484, 1990.

[6] A. Bussmann-Holder, A. R. Bishop, and A. Simon, "Enhancement of Tc in BCS theory extended by interband two-phonon exchange," Zeitschrift für Physik B, vol. 90, no. 2, pp. 183-186, 1993.

[7] B. K. Chakraverty, "Superconductive solutions for a two-band Hamiltonian," Physical Review B, vol. 48, no. 6, pp. 4047-4053, 1993.

[8] A. Bussmann-Holder, A. R. Bishop, H. Büttner, T. Egami, R. Micnas, and K. A. Müller, "The phase diagram of high- $T_{c}$ superconductors in the presence of dynamic stripes," Journal of Physics, vol. 13, no. 23, p. L545, 2001.

[9] A. Bussmann-Holder, A. R. Bishop, H. Büttner et al., Journal of Physics, vol. 13, p. L168, 2001.

[10] A. Bussmann-Holder and R. Micnas, "On the possibility of s+d wave superconductivity within a two-band scenario for high $\mathrm{T}_{c}$ cuprates," Journal of Superconductivity and Novel Magnetism, vol. 15, no. 5, pp. 321-325, 2002.

[11] A. Y. Liu, I. I. Mazin, and J. Kortus, "Beyond Eliashberg superconductivity in $\mathrm{MgB}_{2}$ : anharmonicity, two-phonon scattering, and multiple gaps," Physical Review Letters, vol. 87, no. 8, Article ID 087005, 2001.

[12] H. D. Yang, J.-Y. Lin, H. H. Li et al., "Order parameter of $\mathrm{MgB}_{2}$ : a fully gapped superconductor," Physical Review Letters, vol. 87, no. 16, Article ID 167003, 2001.

[13] X. K. Chen, M. J. Konstantinovi, J. C. Irwin et al., "Evidence for two superconducting gaps in $\mathrm{MgB}_{2}$," Physical Review Letters, vol. 87, Article ID 157002, 2001.
[14] G. C. Rout and S. Das, "Temperature dependence of superconducting gap of heavy fermion system," Physica C, vol. 339, no. 1, pp. 17-26, 2000.

[15] A. Bussmann-Holder, A. Simon, and A. R. Bishop, "Exotic pairing instability in the heavy-fermion superconductor CeCoIn ${ }_{5}$," Europhysics Letters, vol. 75, article 308, 2006.

[16] O. V. Dolgov, R. K. Kremer, J. Kortus, A. A. Golubov, and S. V. Shulga, "Thermodynamics of two-band superconductors: the case of $\mathrm{MgB}_{2}$," Physical Review B, vol. 72, no. 5, Article ID 059902, 2005.

[17] I. I. Mazin, O. K. Andersen, O. Jepsen et al., "Superconductivity in $\mathrm{MgB}_{2}$ : clean or dirty?” Physical Review Letters, vol. 89, no. 10, pp. 10700-10704, 2002.

[18] I. N. Askerzade and B. Tanatar, "Angular dependence of upper critical field in two-band Ginzburg-Landau theory," Physica C, vol. 459, no. 1-2, pp. 56-61, 2007.

[19] A. Changjan and P. Udomsamuthirun, "The critical magnetic field of anisotropic two-band magnetic superconductors," Solid State Communications, vol. 151, no. 14-15, pp. 988-992, 2011.

[20] A. A. Golubov and A. E. Koshelev, "Mixed state in dirty twoband superconductors: application to $\mathrm{MgB}_{2}$," Physica C, vol. 408-410, no. 1-4, pp. 338-340, 2004.

[21] W. A. Fertig, D. C. Johnston, L. E. DeLong, R. W. McCallum, M. B. Maple, and B. T. Matthias, "Destruction of superconductivity at the onset of long-range magnetic order in the compound $\mathrm{ErRh}_{4} \mathrm{~B}_{4}$," Physical Review Letters, vol. 38, no. 17, pp. 987-990, 1977.

[22] M. Ishikawa, O. Fischer, M. Ishikawa, and O. Fischer, "Destruction of superconductivity by magnetic ordering in $\mathrm{Ho}_{1.2} \mathrm{Mo}_{6} \mathrm{~S}_{8}$," Solid State Communications, vol. 23, no. 1, pp. 37-39, 1977.

[23] M. J. Nass, K. Levin, and G. S. Grest, "Bardeen-cooper-schrieffer pairing in antiferromagnetic superconductors," Physical Review Letters, vol. 46, no. 9, pp. 614-617, 1981.

[24] Y. Suzumura and A. D. S. Nagi, "Effect of a homogeneous magnetic field on one-dimensional antiferromagnetic superconductors," Physical Review B, vol. 24, no. 9, pp. 5103-5109, 1981.

[25] M. Ichimura, M. Fujita, and K. Nakao, "Charge-density wave with imperfect nesting and superconductivity," Physical Review $B$, vol. 41, no. 10, pp. 6387-6393, 1990.

[26] G. C. Rout and S. Das, "Temperature dependence of superconducting gap of heavy fermion system," Physica C, vol. 339, no. 1, pp. 17-26, 2000.

[27] S. K. Panda and G. C. Rout, "Interplay of CDW, SDW and superconductivity in high- $\mathrm{T}_{c}$ cuprates," Physica $C$, vol. 469, no. 13, pp. 702-706, 2009.

[28] B. K. Sahoo and B. Panda, "Effect of hybridization and dispersion of quasiparticles on the coexistent state of superconductivity and antiferromagnetism in $\mathrm{RNi}_{2} \mathrm{~B}_{2} \mathrm{C}$, Pramana Journal of Physics, vol. 77, no. 4, pp. 715-726, 2011.

[29] P. Udomsamuthirun, S. Ratanaburi, N. Saentalard, and S. Yoksan, "The ratio $2 \Delta 0 / \mathrm{T}_{c}$ in BCS superconductivity," Journal of Superconductivity, vol. 9, no. 6, pp. 603-604, 1996.

[30] G. C. Rout, M. S. Ojha, and S. N. Behera, "Superconducting gap anomaly in heavy fermion systems," Pramana Journal of Physics, vol. 70, no. 4, pp. 711-730, 2008.

[31] H. Oh, J. Moon, D. Shin, C. Moon, and H. J. Choi, "Brief review on iron-based superconductors: are there clues for unconventional superconductivity?" Progress in Superconductivity, vol. 13, pp. 65-84, 2011.

[32] R. Khasanov, M. Bendele, A. Bussmann-Holder, and H. Keller, "Intrinsic and structural isotope effects in iron-based superconductors," Physical Review B, vol. 82, Article ID 212505, 2010. 
[33] R. Khasanov, M. Bendele, K. Conder, H. Keller, E. Pomjakushima, and V. Pomjakushima, "Iron isotope effect on the superconducting transition temperature and the crystal structure of $\mathrm{FeSe}_{1-x}$, New Journal of Physics, vol. 12, Article ID 073024, 2010. 

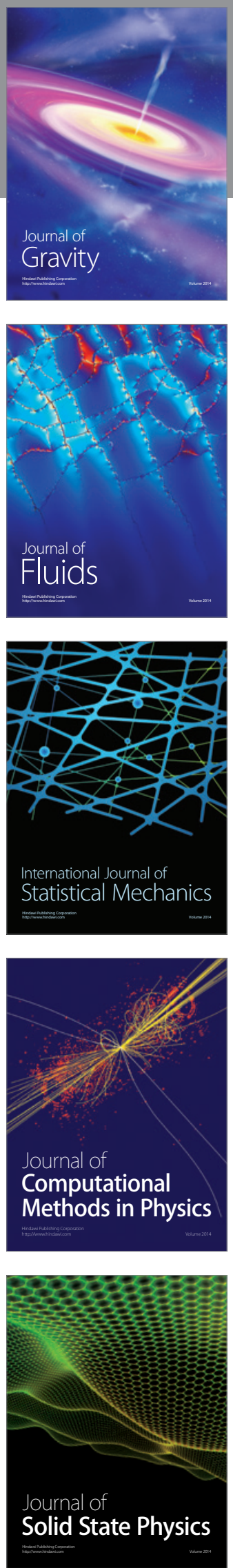

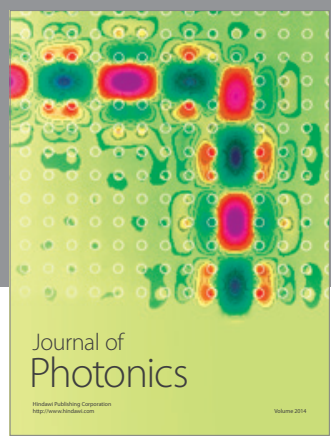

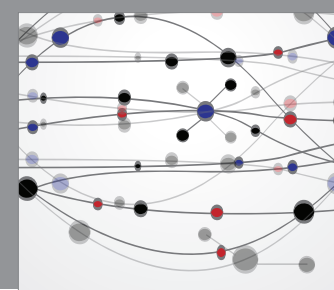

The Scientific World Journal

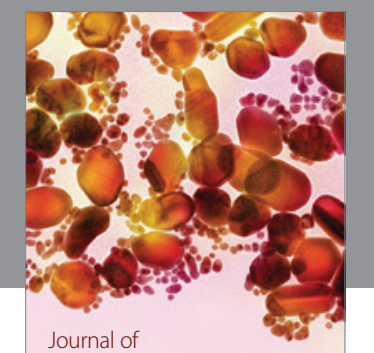

Soft Matter
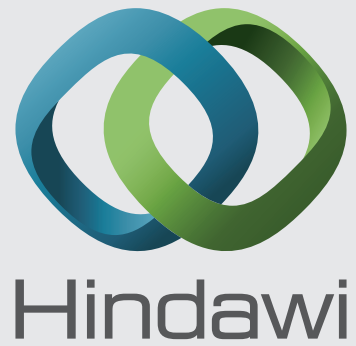

Submit your manuscripts at

http://www.hindawi.com
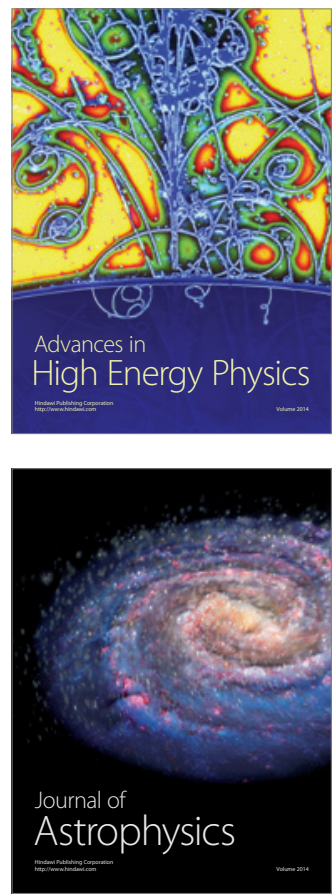
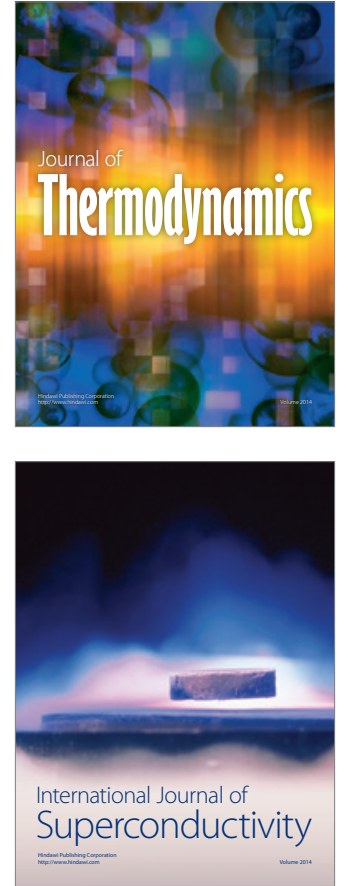
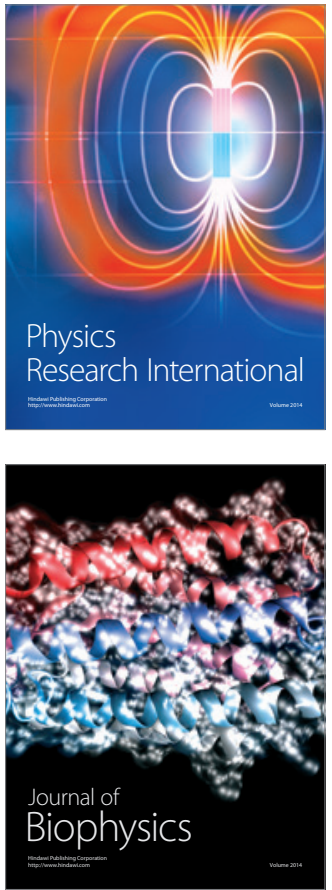
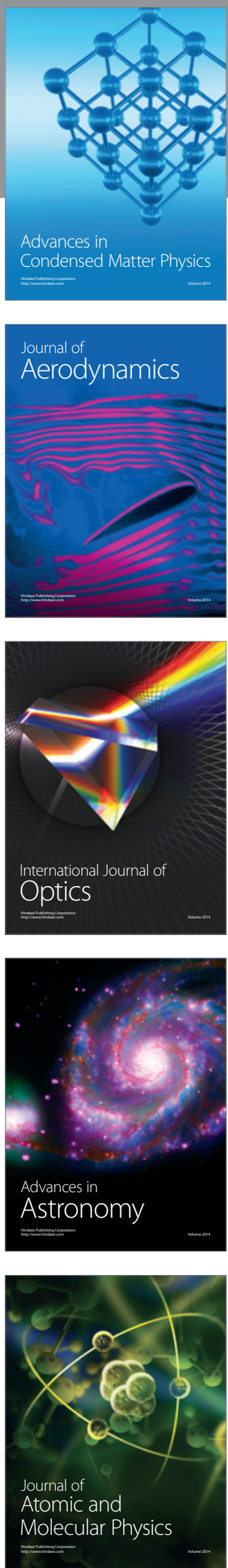